\title{
THE REAL AND RATIONAL COHOMOLOGY OF DIFFERENTIAL FIBRE BUNDLES
}

\author{
BY
}

JOEL WOLF

\begin{abstract}
Consider a differential fibre bundle $(E, \pi, X, G / H, G)$. Under certain reasonable hypotheses, the cohomology of the total space $E$ is computed in terms of the cohomology of the base space $X$ and algebraic invariants of the imbedding of $H$ into $G$.
\end{abstract}

0. Introduction. Consider a differential fibre bundle

$$
\sigma=(E, \pi, X, G / H, G) \text {, }
$$

where $G$ is a compact, connected Lie group and $H$ is a compact, connected subgroup of $G, E$ and $X$ are differentiable manifolds, and $\pi: E \rightarrow X$ is a differentiable map. One would like to compute the cohomology of the total space $E$ in terms of the cohomology of the base space $X$ and certain algebraic invariants of the imbedding of $H$ into $G$. Specifically, there exists a universal bundle

$$
B \sigma(G, H)=(B H, f, B G, G / H, G),
$$

and a classifying diagram

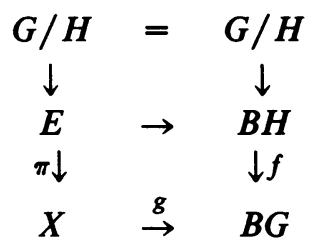

One would like to obtain some sort of isomorphism

$$
H^{*}(E ; K) \cong \operatorname{tor}_{H^{*}(B G ; K)}\left(H^{*}(X ; K), H^{*}(B H ; K)\right),
$$

where $H^{*}(X ; K)$ is regarded as a right $H^{*}(B G ; K)$-module via the multiplicative map $g^{*}$ and $H^{*}(B H ; K)$ is regarded as a left $H^{*}(B G ; K)$-module via the multiplicative map $f^{*}$.

One does have the following result, due to Eilenberg and Moore [4], [5]:

THEOREM 1. Given a differentiable fibre bundle

Received by the editors June 29, 1977.

AMS (MOS) subject classifications (1970). Primary 55F20; Secondary 55H20.

Key words and phrases. Cohomology, fibre bundle, Eilenberg-Moore Spectral Sequence.

() American Mathematical Society 1979 


$$
\sigma=(E, \pi, X, G / H, G),
$$

there exists an algebra isomorphism

$$
\phi: H^{*}(E ; K) \cong \operatorname{Tor}_{C^{*}(B G ; K)}\left(C^{*}(X ; K), C^{*}(B H ; K)\right),
$$

where $C^{*}(X ; K)$ is regarded as a right differential $C^{*}(B G ; K)$-module via the differential multiplicative map $g^{\#}$ and $C^{*}(B H ; K)$ is regarded as a right differential $C^{*}(B G ; K)$-module via the differential multiplicative map $f^{\#}$.

In this paper we prove the following result.

THEOREM. Given a differential fibre bundle

$$
\sigma=(E, \pi, X, G / H, G) \text {, }
$$

where $K$ is the reals $\mathbf{R}$ or the rationals $\mathbf{Q}$, and $X=G^{\prime} / H^{\prime}$ is a homogeneous space formed as the quotient of a compact, connected Lie group $G^{\prime}$ by $a$ compact, connected subgroup $H^{\prime}$ of deficiency 0 in $G^{\prime}$, then there is an algebra isomorphism

$$
H^{*}(E ; K) \cong \operatorname{tor}_{H^{*}(B G ; K)}\left(H^{*}(X ; K), H^{*}(B H ; K)\right) .
$$

This result is a generalization of the results of Baum and Smith [2], and is in the same spirit. See also Wolf [7], [8] for related results.

$\S 1$ contains the necessary preliminaries. $\$ 2$ contains the proof of the Theorem.

1. tor, Tor, and the two-sided Koszul construction. In this section we shall define tor $_{A}(M, N)$ and $\operatorname{Tor}_{A}(M, N)$ in the special case where

$$
A=P\left[x_{1}, \ldots, x_{n}\right]
$$

is a polynomial algebra. The definition is in terms of the so-called two-sided Koszul construction.

Fix $K$ to be a field, and suppose $P\left[x_{1}, \ldots, x_{n}\right]$ is a polynomial algebra over $K$. Consider the exterior algebra

$$
E\left[\mu_{1}, \ldots, \mu_{n}\right]
$$

over $K$, where $\mu_{i}$ has INTERNAL degree $\operatorname{Deg}\left(x_{i}\right)$, EXTERNAL degree -1 , and hence bidegree $\left(\operatorname{Deg}\left(x_{i}\right),-1\right)$ and degree $\operatorname{Deg}\left(x_{i}\right)-1$ in the associated graded algebra over $K$.

(a) tor. Suppose that $A=P\left[x_{1}, \ldots, x_{n}\right], M$ is a right $A$-module, and $N$ is a left $A$-module. We form the complex $M \otimes E\left[\mu_{1}, \ldots, \mu_{n}\right] \otimes N$ with the natural differential $d_{E}$ given by

$$
\begin{aligned}
& d_{E}(m \otimes 1 \otimes n)=0, \\
& d_{E}\left(m \otimes \mu_{i} \otimes n\right)=m x_{i} \otimes 1 \otimes n+m \otimes 1 \otimes x_{i} n, \\
& d_{E} \text { a derivation. }
\end{aligned}
$$


$d_{E}$ is called the EXTERNAL differential, since it acts on external degree. We call the complex

$$
\left(M \otimes E\left(\mu_{1}, \ldots, \mu_{n}\right) \otimes N, d_{E}\right)
$$

the FIRST TWO-SIDED KOSZUL CONSTRUCTION. Observe that the composition $d_{E} \circ d_{E} \equiv 0$. The first two-sided Koszul construction thus has the structure of a differential graded module over $K$.

TheOREM 2. $\operatorname{tor}_{A}(M, N)$ is the homology of the first two-sided Koszul construction:

$$
\operatorname{tor}_{A}(M, N) \cong H\left(M \otimes E\left[\mu_{1}, \ldots, \mu_{n}\right] \otimes N, d_{E}\right) .
$$

Proof. Simply check that the first two-sided Koszul construction is a projective resolution. See, for example, Baum and Smith [2].

(b) Tor. Suppose again that $A=P\left[x_{1}, \ldots, x_{n}\right]$ but now suppose also that $M$ is a right differential $A$-module, and $N$ is a left differential $A$-module. We again form the complex $M \otimes E\left[\mu_{1}, \ldots, \mu_{n}\right] \otimes N$, this time with the natural differential $d_{T}=d_{E}+d_{l}$, where

$$
\begin{aligned}
& d_{I}(m \otimes 1 \otimes n)=d m \otimes 1 \otimes n+(-1)^{\operatorname{Deg}(m)} m \otimes 1 \otimes d n, \\
& d_{I}\left(m \otimes \mu_{i} \otimes n\right)=-d m \otimes \mu_{i} \otimes n-(-1)^{\operatorname{Deg}(m)} m \otimes \mu_{i} \otimes d n, \\
& d_{I} \text { a derivation. }
\end{aligned}
$$

$d_{I}$ is called the INTERNAL differential, since it acts on internal degree. We call the complex

$$
\left(M \otimes E\left[\mu_{1}, \ldots, \mu_{n}\right] \otimes N, d_{T}\right)
$$

the SECOND TWO-SIDED KOSZUL CONSTRUCTION. Observe that the signs have been chosen so that $d_{T} \circ d_{T} \equiv 0$. The second two-sided Koszul construction thus has the structure of a differential graded module over $K$.

Theorem 3. $\operatorname{Tor}_{A}(M, N)$ is the homology of the second two-sided Koszul construction:

$$
\operatorname{Tor}_{A}(M, N) \cong H\left(M \otimes E\left[\mu_{1}, \ldots, \mu_{n}\right] \otimes N, d_{T}\right) .
$$

Proof. Simply check that the second two-sided Koszul construction is a differential projective resolution. See, for example, Baum and Smith [2].

Now let us recall two important naturality results for torsion products. The first is simply functoriality:

THEOREM 4. Consider the following commutative diagram:

$$
\begin{array}{ccccc}
N_{1} & \stackrel{\alpha}{\leftarrow} & A_{1} & \stackrel{\beta}{\rightarrow} & M_{1} \\
f \uparrow & & g \uparrow & & \uparrow h \\
N_{2} & \stackrel{\gamma}{\leftarrow} & A_{2} & \stackrel{\delta}{\rightarrow} & M_{2}
\end{array}
$$


Suppose $A_{1}, A_{2}, M_{1}, M_{2}, N_{1}, N_{2}$ are differential graded algebras over $K$ while $f$, $g, h, \alpha, \beta, \gamma, \delta$ are differential multiplicative maps. Then:

(i) If $g_{*}: H\left(A_{2}\right) \rightarrow H\left(A_{1}\right)$ is an isomorphism, then so is

$$
\operatorname{Tor}_{g}(1,1): \operatorname{Tor}_{A_{2}}\left(M_{1}, N_{1}\right) \rightarrow \operatorname{Tor}_{A_{1}}\left(M_{1}, N_{1}\right) \text {. }
$$

(ii) If $f_{*}: H\left(N_{2}\right) \rightarrow H\left(N_{1}\right)$ is an isomorphism, then so is

$$
\operatorname{Tor}_{1}(1, f): \operatorname{Tor}_{A_{2}}\left(M_{2}, N_{2}\right) \rightarrow \operatorname{Tor}_{A_{2}}\left(M_{2}, N_{1}\right) \text {. }
$$

(iii) If $h_{*}: H\left(M_{2}\right) \rightarrow H\left(M_{1}\right)$ is an isomorphism, then so is

$$
\operatorname{Tor}_{1}(h, 1): \operatorname{Tor}_{A_{2}}\left(M_{2}, N_{2}\right) \rightarrow \operatorname{Tor}_{A_{2}}\left(M_{1}, N_{2}\right) \text {. }
$$

And the second is a comparison theorem:

TheOREM 5. Suppose $P\left[x_{1}, \ldots, x_{n}\right]$ is a polynomial algebra over $K, M$ and $N$ are differential graded algebras over $K$, and $f, g: P\left[x_{1}, \ldots, x_{n}\right] \rightarrow N$ and $h$ : $P\left[x_{1}, \ldots, x_{n}\right] \rightarrow M$ are differential multiplicative maps. If $f$ and $g$ are chain homotopic, then $\operatorname{Tor}_{P\left[x_{1}, \ldots, x_{n}\right]}(M, N)$ is unambiguously defined; that is, $\operatorname{Tor}_{P\left[x_{1}, \ldots, x_{n}\right]}(M, N)$ is the same whether $N$ is regarded as a left differential $P\left[x_{1}, \ldots, x_{n}\right]-$ module via $f$ or via $g$ :

$$
\left(\operatorname{Tor}_{P\left[x_{1}, \ldots, x_{n}\right]}(M, N)\right)_{f} \cong\left(\operatorname{Tor}_{P\left[x_{1}, \ldots, x_{n}\right]}(M, N)\right)_{g} .
$$

An analogous result is true for $\operatorname{Tor}_{P\left[x_{1}, \ldots, x_{n}\right]}(N, M)$ :

$$
\left(\operatorname{Tor}_{P\left[x_{1}, \ldots, x_{n}\right]}(N, M)\right)_{f} \cong\left(\operatorname{Tor}_{P\left[x_{1}, \ldots, x_{n}\right]}(N, M)\right)_{g} .
$$

Proof. We form $M \otimes E\left[\mu_{1}, \ldots, \mu_{n}\right] \otimes N$ with the differential $d_{f}$ obtained via $f$ and with the differential $d_{g}$ obtained via $g$. Now construct the map

$T:\left(M \otimes E\left[\mu_{1}, \ldots, \mu_{n}\right] \otimes N, d_{f}\right) \rightarrow\left(M \otimes E\left[\mu_{1}, \ldots, \mu_{n}\right] \otimes N, d_{g}\right)$

as follows: Since $f$ and $g$ are chain homotopic, there exists, for each $i=$ $1, \ldots, n$, an element $h_{i} \in N$ such that

$$
f\left(x_{i}\right)=g\left(x_{i}\right)-d\left(h_{i}\right) .
$$

Therefore set

$$
\begin{aligned}
T(m \otimes 1 \otimes n) & =m \otimes 1 \otimes n, \\
T\left(1 \otimes \mu_{i} \otimes 1\right) & =1 \otimes \mu_{i} \otimes 1-1 \otimes 1 \otimes h_{i} .
\end{aligned}
$$

We claim that $T$ is a map of differential graded algebras; the proof is a direct calculation:

$$
\begin{aligned}
T d_{f}(m \otimes 1 \otimes n) & =d m \otimes 1 \otimes n+(-1)^{\operatorname{Deg} m} m \otimes 1 \otimes d n \\
& =d_{g} T(m \otimes 1 \otimes n) \\
T d_{f}\left(1 \otimes \mu_{i} \otimes 1\right) & =h\left(x_{i}\right) \otimes 1 \otimes 1+1 \otimes 1 \otimes f\left(x_{i}\right) \\
& =h\left(x_{i}\right) \otimes 1 \otimes 1+1 \otimes 1 \otimes g\left(x_{i}\right)-1 \otimes 1 \otimes d\left(h_{i}\right) \\
& =d_{g}\left(1 \otimes \mu_{i} \otimes 1-1 \otimes 1 \otimes h_{i}\right)=d_{g} T\left(1 \otimes \mu_{i} \otimes 1\right) .
\end{aligned}
$$


Since $T$ has an obvious inverse it follows that $T_{*}$ is the desired isomorphism. The second assertion is proved analogously.

2. The real and rational cohomology of differential fibre bundles. In this section we prove the main theorem. For the sake of exposition, let us first make the following additional assumptions:

(1) $K=\mathbf{R}$.

(2) $H^{\prime}$ has maximal rank in $G^{\prime}$.

The general result then will follow by some elementary remarks at the end of the section.

Notation. If $M$ is a Riemannian manifold modeled on a separable Hilbert space, then we denote by $\mathbf{R}^{\sharp}(M)=\mathbf{R}^{\sharp}(M, d)$ the differential graded algebra of de Rham cochains with exterior derivative. Recall that we have a natural algebra isomorphism

$$
H^{*}(M, \mathbf{R}) \cong H\left(\mathbf{R}^{\sharp}(M, d)\right) .
$$

Consider the following classifying diagrams:

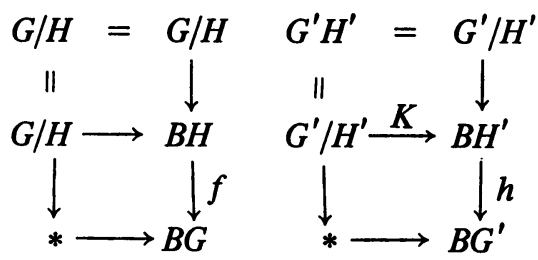

These give rise to the following diagrams in de Rham cochains:

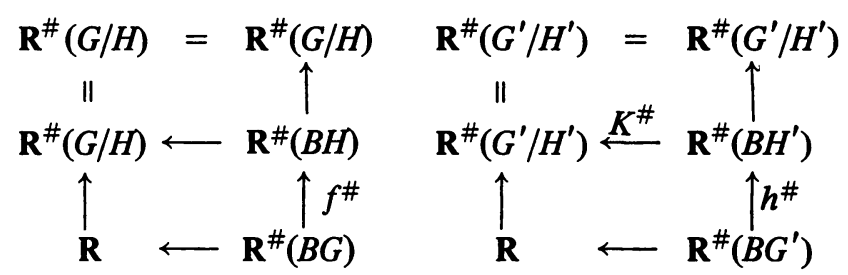

And these, in turn, give rise to the following diagrams in cohomology:

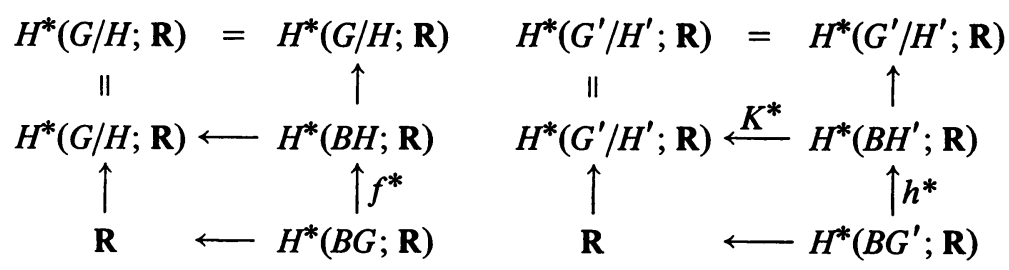

(We may assume that all spaces are differential manifolds modeled after separable Hilbert spaces, and that all maps are differentiable. See, for example, Eells [3].)

We know that $H^{*}(B G ; \mathbf{R}), H^{*}(B H ; \mathbf{R}), H^{*}\left(B G^{\prime} ; \mathbf{R}\right)$, and $H^{*}\left(B H^{\prime} ; \mathbf{R}\right)$ are 
polynomial algebras on generators of even degree. In fact, let

$$
\begin{aligned}
H^{*}(B G ; \mathbf{R}) & =P\left[x_{1}, \ldots, x_{m}\right], & H^{*}\left(B G^{\prime} ; \mathbf{R}\right) & =P\left[z_{1}, \ldots, z_{p}\right], \\
H^{*}(B H ; \mathbf{R}) & =P\left[y_{1}, \ldots, y_{n}\right], & H^{*}\left(B H^{\prime} ; \mathbf{R}\right) & =P\left[w_{1}, \ldots, w_{q}\right] .
\end{aligned}
$$

Let us now recall the relevant facts about maximal rank spaces. Since $\operatorname{Rank}\left(G^{\prime}\right)=p$ and $\operatorname{Rank}\left(H^{\prime}\right)=q$, it follows that $p=q$. It further follows that the sequence

$$
\mathbf{R} \rightarrow H^{*}\left(B G^{\prime} ; \mathbf{R}\right) \stackrel{h^{*}}{\rightarrow} H^{*}\left(B H^{\prime} ; \mathbf{R}\right) \stackrel{K^{*}}{\rightarrow} H^{*}\left(G^{\prime} / H^{\prime} ; \mathbf{R}\right) \rightarrow \mathbf{R}
$$

is coexact. For further details see Baum [1].

Construction. Now choose arbitrary representative cocycles $\mu_{1}, \ldots, \mu_{m}$ in $\mathbf{R}^{\sharp}(B G)$ for $x_{1}, \ldots, x_{m}$, respectively. Define a map $\alpha$ as follows: for each $i=1, \ldots, m$, define $\alpha\left(x_{i}\right)=\mu_{i}$. Since $\mathbf{R}^{\sharp}(B G)$ is graded commutative, the map extends to a unique differential multiplicative map

$$
\alpha: H^{*}(B G ; \mathbf{R}) \rightarrow \mathbf{R}^{\sharp}(B G) \text {. }
$$

From its definition it is clear that $\alpha$ induces the identity map in homology.

We can similarly construct differential multiplicative maps

$$
\begin{aligned}
\beta: H^{*}(B H ; \mathbf{R}) & \rightarrow \mathbf{R}^{\sharp}(B H), \\
\gamma: H^{*}\left(B H^{\prime} ; \mathbf{R}\right) & \rightarrow \mathbf{R}^{\sharp}\left(B H^{\prime}\right), \\
\delta: H^{*}\left(B G^{\prime} ; \mathbf{R}\right) & \rightarrow \mathbf{R}^{\sharp}\left(B G^{\prime}\right),
\end{aligned}
$$

all inducing the identity in homology.

Now consider the following diagram (which we do not claim to be commutative):

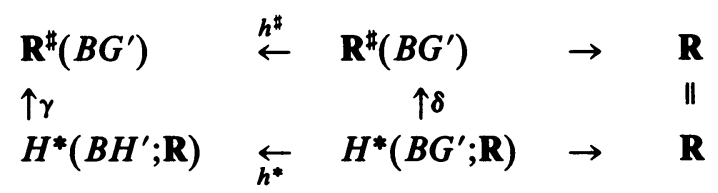

Using the above we are now able to consider the following diagram (which, again, we do not claim to be commutative):

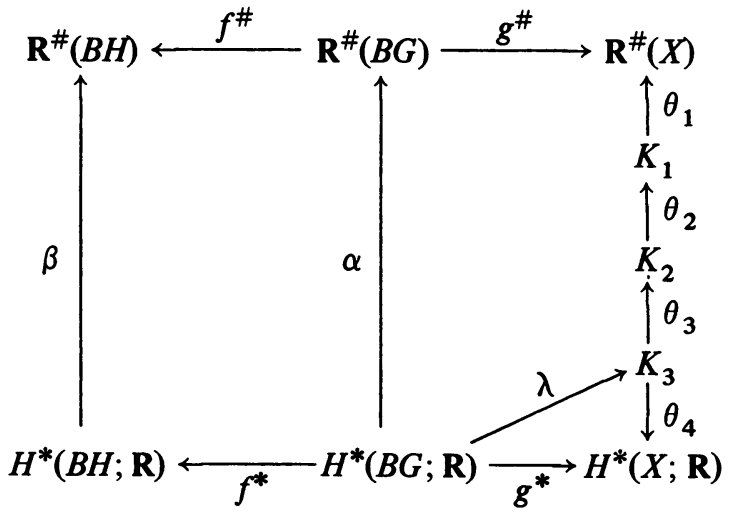


where

(a) We define $K_{1}$ to be the one-sided Koszul construction for computing $7 \operatorname{Tor}_{H^{*}\left(B G^{\prime} ; \mathbf{R}\right)}\left(\mathbf{R}, \mathbf{R}^{\sharp}\left(B H^{\prime}\right)\right)$; in other words,

$$
K_{1}=E\left[\mu_{1}, \ldots, \mu_{p}\right] \otimes \mathbf{R}^{\sharp}\left(B H^{\prime}\right),
$$

where

$$
d\left(\mu_{i} \otimes 1\right)=1 \otimes h^{\sharp} \delta\left(x_{i}\right), \quad d(1 \otimes w)=1 \otimes d(w) .
$$

(b) We define $K_{2}$ to be the one-sided Koszul construction for computing LTor $_{H^{*}\left(B G^{\prime} ; \mathbf{R}\right)}\left(\mathbf{R}, \mathbf{R}^{\sharp}\left(B H^{\prime}\right)\right)$; in other words,

$$
K_{2}=E\left[\mu_{1}, \ldots, \mu_{p}\right] \otimes \mathbf{R}^{\sharp}\left(B H^{\prime}\right),
$$

where

$$
d\left(\mu_{i} \otimes 1\right)=1 \otimes \gamma h^{*}\left(x_{i}\right), \quad d(1 \otimes w)=1 \otimes d(w) .
$$

(c) We define $K_{3}$ to be the one-sided Koszul construction for computing tor $_{H^{*}\left(B G^{\prime} ; \mathbf{R}\right)}\left(\mathbf{R}, H^{*}\left(B H^{\prime} ; \mathbf{R}\right)\right)$, in other words,

$$
K_{3}=E\left[\mu_{1}, \ldots, \mu_{p}\right] \otimes H^{*}\left(B H^{\prime} ; \mathbf{R}\right),
$$

where

$$
d\left(\mu_{i} \otimes 1\right)=1 \otimes h^{*}\left(x_{i}\right), \quad d(1 \otimes w)=0 .
$$

(d) $\theta_{1}$ is defined as follows: To define $\theta_{1}\left(\mu_{i} \otimes 1\right)$, we note that $K^{\sharp} h^{\sharp} \delta\left(x_{i}\right)$ is a coboundary in $\mathbf{R}^{\sharp}(X)$. Therefore choose, for each $i=1, \ldots, p$, an arbitrary element $r_{i} \in \mathbf{R}^{\sharp}(X)$ such that $d\left(r_{i}\right)=K^{\sharp} h^{\sharp} \delta\left(x_{i}\right)$. Now set

$$
\theta_{1}\left(\mu_{i} \otimes 1\right)=r_{i}, \quad \theta_{1}(1 \otimes w)=K^{\sharp}(w) .
$$

The proof that $\theta_{1}$ is a differential multiplicative map is a direct calculation:

$$
\begin{gathered}
d \theta_{1}\left(\mu_{i} \otimes 1\right)=d\left(r_{i}\right)=K^{\sharp} h^{\sharp} \delta\left(x_{i}\right)=\theta_{1}\left(1 \otimes h^{\sharp} \delta\left(x_{i}\right)\right)=\theta_{1} d\left(\mu_{i} \otimes 1\right) . \\
d \theta_{1}(1 \otimes w)=d\left(K^{\sharp}(w)\right)=K^{\sharp}(d(w))=\theta_{1}(1 \otimes d(w))=\theta_{1} d(1 \otimes w) .
\end{gathered}
$$

Observe that $\theta_{1}$ induces an isomorphism in homology.

(e) $\theta_{2}$ is defined to be the differential multiplicative map which induces the identity in homology given by Theorem 5 ; in other words,

$$
\theta_{2}\left(\mu_{i} \otimes 1\right)=\mu_{i} \otimes 1-1 \otimes S_{i}, \quad \theta_{2}(1 \otimes w)=1 \otimes w,
$$

where $S_{i} \in \mathbf{R}^{\sharp}\left(B H^{\prime}\right)$ is such that $\gamma h^{*}\left(x_{i}\right)=h^{\sharp} \delta\left(x_{i}\right)-d\left(S_{i}\right)$.

(f) $\theta_{3}$ is defined as follows:

$$
\theta_{3}\left(\mu_{i} \otimes 1\right)=\mu_{i} \otimes 1, \quad \theta_{3}(1 \otimes w)=1 \otimes \gamma(w) .
$$

The proof that $\theta_{3}$ is a differential multiplicative map is a direct calculation: 


$$
\begin{aligned}
d \theta_{3}\left(\mu_{i} \otimes 1\right) & =d\left(\mu_{i} \otimes 1\right)=1 \otimes \gamma h^{*}\left(x_{i}\right) \\
& =\theta_{3}\left(1 \otimes h^{*}\left(x_{i}\right)\right)=\theta_{3} d\left(\mu_{i} \otimes 1\right) . \\
d \theta_{3}(1 \otimes w) & =d(1 \otimes \gamma(w))=1 \otimes d(\gamma(w)) \\
& =1 \otimes \gamma(d(w))=0 \\
& =\theta_{3}(0)=\theta_{3} d(1 \otimes w) .
\end{aligned}
$$

Observe that $\theta_{3}$ induces an isomorphism in homology.

(g) $\theta_{4}$ is defined as follows:

$$
\theta_{4}\left(\mu_{i} \otimes 1\right)=0, \quad \theta_{4}(1 \otimes w)=K^{*}(w) .
$$

The proof that $\theta_{4}$ is a differential multiplicative map is a direct calculation:

$$
\begin{gathered}
d \theta_{4}\left(\mu_{i} \otimes 1\right)=d(0)=0=K^{*} h^{*}\left(x_{i}\right)=\theta_{4}\left(1 \otimes h^{*}\left(x_{i}\right)\right)=\theta_{4} d\left(\mu_{i} \otimes 1\right) . \\
d \theta_{4}(1 \otimes w)=d\left(K^{*}(w)\right)=0=\theta_{4}(0)=\theta_{4} d(1 \otimes w) .
\end{gathered}
$$

(h) We construct a differential multiplicative map

$$
\lambda: H^{*}(B G ; \mathbf{R}) \rightarrow K_{3}
$$

which induces $g^{*}$ in homology, by analogy with the maps $\alpha, \beta, \gamma, \delta$ above. Choose arbitrary cocycles $f_{1}, \ldots, f_{m} \in K_{3}$ representing $g^{*}\left(x_{1}\right), \ldots, g^{*}\left(x_{m}\right)$, respectively. Set $\lambda\left(z_{i}\right)=t_{i}$ and extend.

Now consider the extreme right-hand side of the diagram. We claim that $\theta_{1} \theta_{2} \theta_{3}$ and $\theta_{4}$ induce the same map in homology. In other words, we claim commutativity in the diagram

$$
\begin{array}{r}
H^{*}(X ; \mathbf{R}) \\
\uparrow \theta_{1 *} \\
H^{*}(X ; \mathbf{R}) \\
\uparrow \theta_{2 *} \\
H^{*}(X ; \mathbf{R}) \\
\uparrow \theta_{3_{*}} \\
H^{*}(X ; \mathbf{R})
\end{array} \theta_{4_{*}}
$$

To see this we examine the effect of applying the maps $\theta_{1} \theta_{2} \theta_{3}$ and $\theta_{4}$ to a cycle in $K_{3}$. A cycle in $K_{3}$ has the form $1 \otimes w$. Now

$$
\theta_{1} \theta_{2} \theta_{3}(1 \otimes w)=\theta_{1} \theta_{2}(1 \otimes \gamma(x))=\theta_{1}(1 \otimes \gamma(w))=K^{\sharp} \gamma(w) ;
$$

on the other hand

$$
\theta_{4}(1 \otimes w)=K^{*}(w)
$$

Thus $\left(\theta_{1} \theta_{2} \theta_{3}\right)_{*}([1 \otimes w])=\left(K^{\sharp} \gamma\right)_{*}([w])=K^{*}([w])=\theta_{4_{*}}([1 \otimes w])$. So the diagram commutes. 
By the definition of $\lambda$ we know that the following diagram is also commutative:

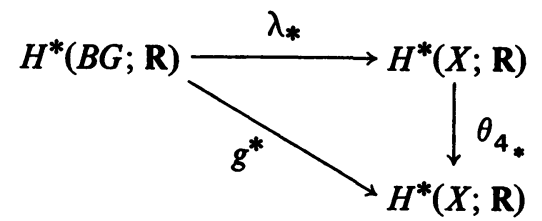

Thus we can extrapolate commutativity in the following diagram:

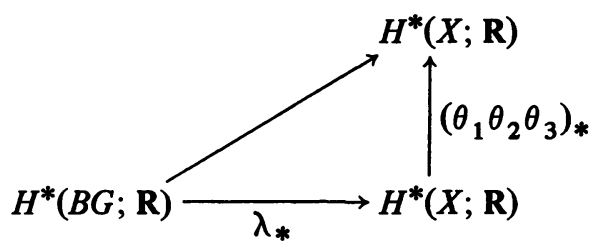

Ultimately we have pieced together the fact that our entire original diagram commutes upon passage to homology. Thus utilizing Theorems 1, 4 and 5 we have the following string of algebra isomorphisms.

$$
\begin{aligned}
& H^{*}(E ; \mathbf{R}) \\
& \cong \emptyset \\
& \operatorname{Tor}_{\mathbf{R}}^{\#(B G)}\left(\mathbf{R}^{\#}(X), \mathbf{R}^{\#}(B H)\right) \\
& \cong \uparrow \operatorname{Tor}_{\alpha}(1,1) \\
& \urcorner \operatorname{Tor}_{H^{*}(B G ; \mathbf{R})}\left(\mathbf{R}^{\#}(X), \mathbf{R}^{\#}(B H)\right) \Gamma \\
& \cong \downarrow T_{*} \\
& \left\lfloor\operatorname{Tor}_{H^{*}}(B G ; \mathbf{R})\left(\mathbf{R}^{\#}(X), \mathbf{R}^{\#}(B H)\right) \Gamma\right. \\
& \cong \downarrow \operatorname{Tor}_{1}(1, \beta) \\
& \operatorname{Tor}_{H^{*}(B G ; \mathrm{R})}\left(\mathrm{R}^{\#}(X), H^{*}(B H ; \mathbf{R})\right) \Gamma \\
& \cong \downarrow U_{*} \\
& \left.\operatorname{Tor}_{H^{*}(B G ; \mathbf{R})}\left(\mathbf{R}^{\#}(X), H^{*}(B H ; \mathbf{R})\right)\right\} \\
& \cong \uparrow \operatorname{Tor}_{1}\left(\theta_{1}, 1\right) \\
& \left.\operatorname{Tor}_{H^{*}(B G ; \mathrm{R})}\left(K_{1}, H^{*}(B H ; \mathbf{R})\right)\right\} \\
& \cong \uparrow \operatorname{Tor}_{1}\left(\theta_{2}, 1\right) \\
& \operatorname{Tor}_{H^{*}(B G ; \mathrm{R})}\left(K_{2}, H^{*}(B H ; \mathrm{R})\right) \\
& \cong \uparrow \operatorname{Tor}_{1}\left(\theta_{3}, 1\right) \\
& \operatorname{Tor}_{H^{*}(B G ; \mathrm{R})}\left(K_{3}, H^{*}(B H ; \mathrm{R})\right) \nearrow \\
& \cong \downarrow \operatorname{Tor}_{1}\left(\theta_{4}, 1\right) \\
& \operatorname{Tor}_{H^{*}(B G ; \mathbf{R})}\left(H^{*}(X ; \mathbf{R}), H^{*}(B H ; \mathbf{R})\right) \curlywedge \\
& =\downarrow \\
& \operatorname{tor}_{H^{*}(B G ; \mathrm{R})}\left(H^{*}(X ; \mathbf{R}), H^{*}(B H ; \mathbf{R})\right)
\end{aligned}
$$


To extend to the deficiency 0 case, recall that the sequence

$$
H^{*}\left(B G^{\prime} ; \mathbf{R}\right) \stackrel{h^{*}}{\rightarrow} H^{*}\left(B H^{\prime} ; \mathbf{R}\right) \stackrel{K^{*}}{\rightarrow} H^{*}\left(G^{\prime} / H^{\prime}\right)
$$

will be coexact. For the definition of deficiency and further details see Baum [1].

The proof goes through as before except that $\theta_{4}$ must be redefined. Write $K_{3}$ as

$$
K_{3}=E\left[\mu_{1}, \ldots, \mu_{s}\right] \otimes E \otimes H^{*}\left(B H^{\prime} ; \mathbf{R}\right)
$$

where the elements $\mu_{1}, \ldots, \mu_{s}$ are not cycles, and the elements of $E$ are. Define

$$
\begin{aligned}
& \theta_{4}\left(\mu_{i} \otimes 1 \otimes 1\right)=0, \\
& \theta_{4}(1 \otimes 1 \otimes w)=K^{*}(w), \\
& \theta_{4}(1 \otimes w \otimes 1)=\left[\theta_{1} \theta_{2} \theta_{3}(1 \otimes w \otimes 1)\right] .
\end{aligned}
$$

The first diagram in our chase is still commutative, this time by our choice of $\theta_{4}$, and the rest of the argument is the same.

Finally we remark that the case $K=\mathbf{Q}$ works equally well by simply using graded commutative rational cochains. See Sullivan [6].

\section{REFERENCES}

1. P. F. Baum, On the cohomology of homogeneous spaces, Topology 7 (1968), 15-38.

2. P. F. Baum and L. Smith, The real cohomology of differentiable fibre bundles, Comment. Math. Helv. 42 (1967), 171-179.

3. J. Eells, A setting for global analysis, Bull. Amer. Math. Soc. 72 (1966), $751-807$.

4. S. Eilenberg and J. C. Moore, Limits and spectral sequerices, Topology 1 (1962), 1-24.

5. , Homology and fibrations. I, Comment. Math. Helv. 40 (1966), 199-236.

6. D. Sullivan, Infinitesimal computations in topology, (to appear).

7. J. Wolf, The cohomology of homogeneous spaces and related topics, Thesis, Brown University, 1973.

8. The cohomology of homogeneous spaces, Amer. J. Math. 97 (1977), 312-340.

Department of Mathematics, Harvard University, Cambridge, Massachusetts 02138

Current address: Bell Laboratories, 3F623A, Crawfords Corner Road, Holmdel, New Jersey 07733 